\title{
Brodie abscess of the jaw: An unusual presentation of a rare complication of subacute osteomyelitis
}

\author{
Melroy D'souza ${ }^{* 1}$, Jack Xu², Elizabeth John², Payal Parikh ${ }^{2}$ \\ ${ }^{1}$ Rutgers Robert Wood Johnson Medical School, United States \\ ${ }^{2}$ Department of Medicine, Rutgers Robert Wood Johnson Medical School, United States
}

Received: July 4, 2017

DOI: $10.5430 /$ crim.v4n3p75
Accepted: August 14, 2017

Online Published: August 28, 2017

\begin{abstract}
Background: A Brodie abscess is a rare subtype of subacute osteomyelitis which can be acquired from local trauma or hematogenous spread secondary to transient bacteremia. However, to date there have been no reports of the presence of a Brodie abscess in the mandible.

Case representation: Here we report a case of a 51-year-old female who presented to the emergency department with a 4-day history of left sided facial pain and trismus after recent dental manipulation. A computed tomography (CT) scan showed the presence of osteomyelitis with a Brodie abscess in the mandibular condyle. Empiric treatment was initiated with ampicillinsulbactam. After seven days of treatment, the antibiotics were switched to ceftriaxone and metronidazole on discharge. Five days later the patient returned to the hospital with the same symptoms that she initially presented with, indicating a failure of treatment. Follow-up CT scan showed the persistence of the Brodie abscess. Subsequently, IV ertapenem was initiated for a duration of eight weeks and the patient was instructed to obtain an oral surgery evaluation as an outpatient.

Conclusions: This case highlights a unique presentation of a Brodie abscess, which has been previously exclusively found in long bones, and raises awareness of such a complication in patients whose facial erythema, pain, and swelling may not improve with first line therapy for a simple dental infection. Additionally, we highlight that the unique location of the mandibular condyle poses a treatment challenge and surgical replacement of the jaw appears to be the lone curative modality.
\end{abstract}

Key Words: Brodie abscess, Jaw osteomyelitis

\section{INTRODUCTION}

Sir Benjamin Collins Brodie described a unique type of abscess in 1832. ${ }^{[1]}$ A Brodie abscess is a collection that forms as a subacute infection of intermedullary bone which can remain asymptomatic for months to years before causing a frank osteomyelitis. ${ }^{[2]}$ The lesion typically forms secondary to a contiguous spread from local trauma or via a hematogenous route from transient bacteremia. ${ }^{[3]}$ This condition is more prevalent in children and it has a predilection for the metaphysis of long bones such as the femur and tibia; when present in adults it is often localized to the diaphysis. ${ }^{[4]}$ The most common offending pathogen is Staphylococcus aureus, although cases with Pseudomonas aeruginosa, Klebsiella spp., and Salmonella typhi have also been reported. ${ }^{[5]}$

Here we present the first case of a Brodie abscess located in the mandibular condyle of a middle-aged woman.

\section{Case presentation}

A 51-year-old female with a history of dental caries treated with restorative fillings and a recent dental cleaning presented

*Correspondence: Melroy D’souza; Email: msd184@rwjms.rutgers.edu; Address: Rutgers Robert Wood Johnson Medical School, United States. 
to our facility with a six-day history of left sided facial pain, swelling, erythema, and trismus. Symptom onset was acute and was accompanied by a subjective fever and odynophagia. She self-administered amoxicillin $500 \mathrm{mg}$ at symptom onset and was switched to cephalexin by her primary medical doctor for suspected sialadenitis at the time. The patient reported no past medical issues, including no history of diabetes as well as no tobacco or illicit substance use.

After she experienced no alleviation of the facial pain, she presented to the emergency department for worsening progression of symptoms. On examination, there was a $3 \mathrm{~cm}$ $\times 4 \mathrm{~cm}$ area of erythema and exquisite tenderness, extending from the tragus of the left ear to the lateral border of the mouth. Initial vital signs were stable with no fever and no leukocytosis. A computed tomography (CT) scan with contrast of the facial bones showed the presence of a Brodie abscess and osteomyelitis in the left mandibular condyle along with abscesses in the adjacent soft tissues (see Figure 1); additionally, there was an enhancement of the temporomandibular joint (TMJ), concerning for septic arthritis. A CT guided drainage of one soft tissue abscess was performed, after which the aspirate and blood were sent for cultures.

Empiric treatment with intravenous (IV) ampicillin and sulbactam was initiated to cover for gram positive and gramnegative bacteria, along with the anaerobes that typically colonize the oropharynx. The patient experienced significant relief of symptoms over the subsequent days with a complete resolution of the facial pain and trismus on the seventh day of antibiotic treatment. At this time the patient remained afebrile, with a normal white blood cell count, and blood as well as aspirate cultures failed to grow organisms; therefore, the decision was made to discharge the patient. Outpatient administration of IV ampicillin-sulbactam required a pump which was not a feasible option for the patient. The oral formulation was thought to have poor penetration into the bone, so it was not used. Therefore, the regimen was switched to IV ceftriaxone and metronidazole for relative ease of at-home administration and the treatment was prescribed for six total weeks of antibiotic therapy.

Four days after discharge, the patient returned to the emergency department with recurrent symptoms of left facial pain and swelling over the same area that she initially presented with. She was compliant with the antibiotics and did not undergo a dental procedure or sustain any form of facial trauma since her discharge. The examination was similar to the initial visit with tenderness, swelling, and erythema over the left face; she remained afebrile with a slightly elevated white blood cell count of $10,300 / \mathrm{mm}^{3}$. Follow-up CT of the face was grossly unchanged. It was determined that the recurrence of symptoms was a result of treatment failure of ceftriaxone and metronidazole. Therapy was switched to IV ertapenem for a duration of eight weeks and the patient was instructed to follow up with an oral surgeon as an outpatient.

This patient received 8 total weeks of ertapenem therapy and was switched to oral augmentin for 10 more weeks prior to undergoing a jaw reconstruction surgery; this procedure was deemed to be the only definitive treatment after evaluation by an oral surgeon.

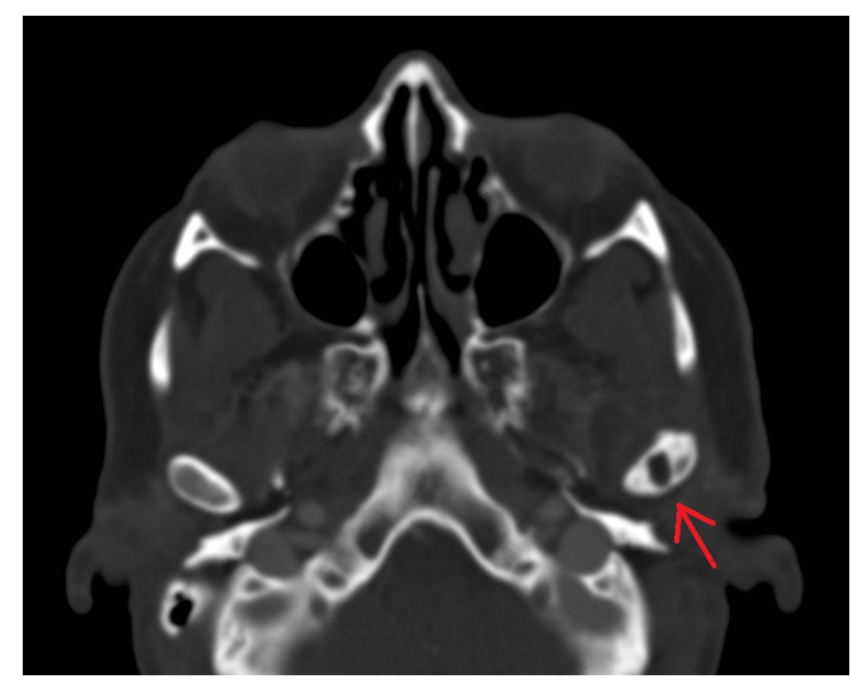

Figure 1. Axial CT scan w/contrast of facial bones

As shown in Figure 1, there is evidence of the formation of an abscess in the left mandibular condyle (red arrow). The lytic lesion in the medullary portion of the bone is pathognomonic for a Brodie abscess. ${ }^{[2]}$

\section{Discussion}

In this report we describe an atypical presentation of a Brodie abscess located in the mandibular condyle. The abscess formation follows an indolent course and is typically discovered as an incidental finding or when it leads to fulminant osteomyelitis. It classically forms secondary to local trauma, which is the most probable etiology in this patient. She underwent several invasive dental procedures over the past 15 years and it is likely that the abscess developed somewhere along the course of her dental work.

The abscess' location in the mandible posed a challenge for treatment and infection eradication. Brodie abscesses have been shown to respond well to both surgical debridement and antibiotic therapy. ${ }^{[6]}$ The structural arrangement of the jaw makes it inaccessible to procedures such as curettage, thereby eliminating the opportunity of a minimally invasive surgical intervention. Furthermore, a consultation with 
an oral surgeon revealed that there was no opportunity for surgical debridement for this patient. Rather the most curative approach would have to include mandibular or TMJ replacement given that the jaw's integrity was markedly compromised and it would likely continue to serve as a nidus for future infections. This assertion was consistent with the findings of Chattopadhyay et al. that condylectomy was the most frequently adopted and most effective treatment of condylar osteomyelitis. ${ }^{[7]}$ Due to her active infection, the patient was not a surgical candidate at the initial visit; therefore, systemic antibiotic therapy was subsequently started.

The mandible is characterized as an irregular type of bone with poor vascularity relative to long bones, which creates a significant obstacle for localized delivery of antimicrobial therapy. Research by Dym and Zeidan cautions against empiric antibiotic therapy due to the prevalence of microbe resistance against commonly used antibiotics, while Krayer, Leite and Kirkwood suggest that initiating treatment prior to receiving the results of cultures and sensitivities is appropriate. ${ }^{[5,8]}$ Our patient was treated with two different antibiotics prior to presentation and arrival at the appropriate diagnosis, which is perhaps why the blood and wound cultures remained negative after several days; this phenomenon is relatively common in other reported cases. ${ }^{[7]}$ While awaiting the results of the cultures, treatment with ampicillin and sulbactam was initiated after reviewing the medication's documented efficacy in cases of jaw osteomyelitis. ${ }^{[9]}$ Other therapeutic options include penicillins, cephalosporins, metronidazole, clindamycin, vancomycin, ertapenem, and carbapenems. ${ }^{[5]}$ There continues to be no consensus about the duration of antibiotic therapy for jaw osteomyelitis, with recommendations ranging from four to eight weeks. ${ }^{[5]}$ In this case we elected to carry out the initial treatment with ertapenem for eight weeks and then switched to oral augmentin leading up to the jaw reconstruction procedure.

\section{Conclusion}

This case highlights the first presentation of a Brodie abscess in the mandibular condyle. Given that the abscess typically forms in the femur and tibia, our clinical suspicion was low for the presence of this form of osteomyelitis. The diagnosis was entirely established by the pathognomonic radiographic finding of a lytic lesion in the condyle with an intraosseous abscess. This case illustrates the importance of using radiologic findings to ascertain a diagnosis, especially when the disease entity is quite uncommon. Therefore, we encourage practitioners to maintain a high index of suspicion when a proposed oral infection does not resolve with initial antibiotic therapy.

A consensus has not been reached regarding the optimal treatment for jaw osteomyelitis. Moreover, given that a Brodie abscess has never emerged in the mandible before, there are no recommendations in the literature to guide management. Our therapy was designed to attenuate the acute infection with the use broad spectrum IV antibiotics, in order to eventually perform surgery to replace the mandibular condyle and restore the integrity of the jaw. This case further highlights that a Brodie abscess of the jaw is a separate entity as compared to jaw osteomyelitis, and requires additional treatment considerations. While osteomyelitis of the jaw can be definitively treated with antibiotics alone, the formation of a Brodie abscess is indicative of structural compromise which requires a reconstruction for complete eradication.

We look forward to future reports of a Brodie abscess presenting in various other atypical locations. For cases of a Brodie abscess located in the jaw, we recommend attenuating the infection with IV antibiotics in order to stabilize the patient for an eventual, and curative, jaw reconstruction surgery.

\section{CONFLicts OF INTEREST Disclosure}

The authors have declared no conflicts of interest.

\section{REFERENCES}

[1] Brodie BC. An Account of Some Cases of Chronic Abscess of the Tibia. Journal of the Royal Society of Medicine. 1832; 17: 239-49. https://doi.org/10.1177/095952873201700111

[2] Guermazi A, Mohr A, Genant HK. Brodie abscess: another type of chronic posttraumatic osteomyelitis. European Radiology. $2003 \mathrm{Feb}$ 27; 13(7): 1750-2. PMid:12835993 https://doi.org/10.1007/ s00330-002-1767-6

[3] Abdulhadi M, White A, Pollock A. Brodie Abscess. Pediatric Emergency Care. 2012; 28(11): 1249-51. PMid:23128659 https://doi . org/10.1097/PEC.0b013e31z8272c561

[4] Ogbonna O, Paul Y, Nabhani H, et al. Brodie's Abscess in a Patient Presenting with Sickle Cell Vasoocclusive Crisis. Case Reports in
Medicine. 2015; 2015: 1-3. PMid:26290668 https://doi.org/ $10.1155 / 2015 / 429876$

[5] Dym H, Zeidan J. Microbiology of Acute and Chronic Osteomyelitis and Antibiotic Treatment. Dental Clinics of North America. 2017; 61(2): 271-82. PMid:28317566 https://doi.org/10.1016/j. cden.2016.12.001

[6] Olasinde A, Oluwadiya K, Adegbehingbe O. Treatment of Brodie's abscess: Excellent results from curettage, bone grafting and antibiotics. Singapore Medical Journal. 2011; 52(6): 436-9. PMid:21731997

[7] Chattopadhyay P, Nagori S, Menon R, et al. Osteomyelitis of the Mandibular Condyle: A Report of 2 Cases With Review of the Literature. Journal of Oral and Maxillofacial Surgery. 2017; 75(2): 322-35. 
PMid:27649464 https://doi.org/10.1016/j.joms.2016.08 .018

[8] Krayer J, Leite R, Kirkwood K. Non-Surgical Chemotherapeutic Treatment Strategies for the Management of Periodontal Diseases. Dental Clinics of North America. 2010; 54(1): 13-33. PMid:20103470 https://doi.org/10.1016/j.cden.2009.08
.010

[9] Baur D, Altay M, Flores-Hidalgo A, et al. Chronic Osteomyelitis of the Mandible: Diagnosis and Management-An Institution's Experience Over 7 Years. Journal of Oral and Maxillofacial Surgery. 2015; 73(4): 655-65. PMid:25577460 https://doi .org/10.101 $6 / j \cdot j o m s .2014 .10 .017$ 